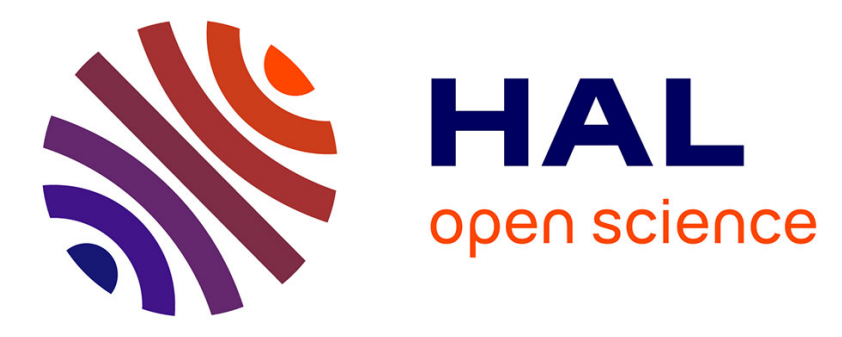

\title{
Smart Directional Data Aggregation in VANETs
}

Sabri Allani, Richard Chbeir, Taoufik Yeferny, Sadok Ben Yahia

\section{To cite this version:}

Sabri Allani, Richard Chbeir, Taoufik Yeferny, Sadok Ben Yahia. Smart Directional Data Aggregation in VANETs. 32nd IEEE International Conference on Advanced Information Networking and Applications, AINA 2018, Krakow, Poland, May 16-18, 2018, May 2018, Krakow, Poland. pp.63-70, 10.1109/AINA.2018.00022 . hal-01908063

\section{HAL Id: hal-01908063 \\ https: / hal-univ-pau.archives-ouvertes.fr/hal-01908063}

Submitted on 14 Jan 2020

HAL is a multi-disciplinary open access archive for the deposit and dissemination of scientific research documents, whether they are published or not. The documents may come from teaching and research institutions in France or abroad, or from public or private research centers.
L'archive ouverte pluridisciplinaire HAL, est destinée au dépôt et à la diffusion de documents scientifiques de niveau recherche, publiés ou non, émanant des établissements d'enseignement et de recherche français ou étrangers, des laboratoires publics ou privés. 


\title{
Smart Directional Data Aggregation in VANETs
}

\author{
Sabri Allani ${ }^{1,2}$, Richard Chbeir $^{2}$, Taoufik Yeferny ${ }^{1}$, and Sadok Ben Yahia ${ }^{1}$ \\ ${ }^{1}$ University of Tunis El Manar, Faculty of Sciences of Tunis, LIPAH-LR11ES14, 2092 Tunis, Tunisia \\ Email: \{sabri.allani, yeferny.taoufik\}@gmail.com sadok.benyahia@fst.rnu.tn \\ ${ }^{2}$ Univ. Pau \& Pays Adour, LIUPPA, 64600 Anglet, France \\ Email: rchbeireacm.org
}

\begin{abstract}
The ultimate goal of a Traffic Information System (TIS) consists in properly informing vehicles about road traffic conditions in order to reduce traffic jams and consequently $\mathrm{CO} 2$ emission while increasing the user comfort. Therefore, the design of an efficient aggregation protocol that combines correlated traffic information like location, speed and direction known as Floating Car Data (FCD) is of paramount importance. In this paper, we introduce a new TIS data aggregation protocol called Smart Directional Data Aggregation (SDDA) able to decrease the network overload while obtaining high accurate information on traffic conditions for large road sections. To this end, we introduce three levels of messages filtering: (i) filtering all FCD messages before the aggregation process based on vehicle directions and road speed limitations, (ii) integrating a suppression technique in the phase of information gathering in order to eliminate the duplicate data, and (iii) aggregating the filtered FCD data and then disseminating it to other vehicles. The performed experiments show that the SDDA outperforms existing approaches in terms of effectiveness and efficiency.

Index Terms-VANET , Traffic Information System (TIS) , Aggregation, Direction, Speed, Floating Car data (FCD)
\end{abstract}

\section{INTRODUCTION}

Vehicular Ad hoc Networks (VANETs) are a specific type of Mobile Ad hoc Networks (MANETs) [1] where each network node, representing mainly a vehicle, can communicate with other vehicles or an existing infrastructure, e.g., Road Side Units (RSU). Recently, there has been a rapid evolution of the technology required for supporting the VANETs. In fact, this technology has been adopted and supported by a lot of pioneering automobile manufacturers [1] (e.g. the US Department of Transportation (DoT), Toyota and Honda etc). This support has participated to the emergence of new ideas for VANET-based applications related to entertainment, safety and non safety information. Entertainment applications include multimedia streaming, P2P file sharing and Internet access [2]. Informational applications include all what concerns the notification of upcoming roadway hazards and traffic conditions, in addition to the collection and dissemination of weather information [2]. The safety applications have been the US DoT's prime focus efforts in vehicular networks including both assistance and collision warning [1]. Intelligent Transportation System (ITS) standards perform communication in VANETs so as to support transportation and cooperation services [1]. Advanced technologies (e.g., image and gesture recognition) have been incorporated to improve information exchanging and ITS effectiveness. As far as the development in the wireless communication field is generally concerned, the ITS applications are developed on the basis of car-tocar communication standards (i.e., Dedicated Short Range Communications (DSRC) and Wireless Access in Vehicular Environment (WAVE)) [1]. Also, the technology of Inter Vehicle Communication (IVC) is enabled for most applications, namely entertainment, enhanced driving, and active safety. Subsequently, it is a must to manage the available limitedbandwidth carefully and efficiently as it is a scarce resource. Thus, communication VANET protocols need a careful design. Hence, to cope with the limited bandwidth, the various requirements emerging from the applications themselves and the highly dynamic network topology. Particularly, the VANETbased applications requiring information gathering and aggregation (e.g., traffic information systems, weather information systems, parking spaces and travel time predictions) have to deal with the following challenges:

1) How to decide if two or more FCD messages must be aggregated or not?

2) How to select timely data to be aggregated (since not all data need to be collected after a certain time)?

3) How to consider data from far vehicles?

4) How to filter the unnecessary and duplicated FCD messages in order to avoid affecting the accuracy of the shared traffic information?

5) How to take into consideration the road traffic signals and speed limitations?

Several data aggregation techniques have been proposed in the literature $[3,4,5,6]$. However, in a high complex urban and highway network, the large amount of traffic information needs smart filtering and selection criteria after the aggregation process. However, all existing techniques mainly focus on combining the correlated items but none of them tries to use a suppression technique in order to eliminate the duplicated messages. To cope with these aforementioned requirements, we introduce here a new aggregation protocol called Smart Directional Data Aggregation (SDDA) that can deal proprely with traffic information in both urban and highway conditions. It consists in properly selecting relevant FCD messages that should be aggregated. To do so, SDDA provides three filters: (i) The first uses vehicle directions. In fact, each vehicle will aggregate only data that corresponds to its direction but only store, carry and forward uninteresting data. (ii) The 
second is done through road speed limitations, thus, if the average speed of the received FCD messages is higher than the maximum allowed speed then it will be ignored and replaced by the maximum allowed speed value. Doing so, we increase the aggregation accuracy. (iii) The last is performed is by integrating a suppression technique [7] in order to eliminate the duplicated inputs in the aggregation phase by providing a high accurate time slot based on the distance and the range that all vehicles should wait before rebroadcasting the FCD message. The rest of the paper is organized as follows. Section II describes the pioneering literature approaches to highlight their drawbacks regarding the aforementioned challenges. In Section III, we describe our proposed protocol for smart data aggregation. Section IV is dedicated to present the simulation settings as well as the evaluation of our suggested approach. The last section concludes this paper and pins down several future directions.

\section{RELATED WORK}

In larger road networks, urban and highway, there is a huge amount of traffic information that quickly exceeds reasonable limits. In this respect, an appropriate aggregation mechanism can reduce the communication cost while obtaining useful aggregated information. Several related approaches have been proposed in the literature.

In [8], the authors put forward Self-Organizing Traffic Informational Systems (SOTIS) where vehicles would periodically exchange their speed and position on the road. Each vehicle in SOTIS would calculate average speeds from neighboring vehicles before rebroadcasting the aggregated data. It would basically provide an outline of the current movement conditions through the use of periodic road information broadcasting. On the one hand, the main SOTIS limitation was related to the broadcast of the periodic Floating Car Data (FCD) since it did not ensure or even indicate how duplicated [FCD] messages that came from the same road segment could be aggregated together. On the other hand, the aggregation of the same duplicated FCD messages would reduce the accuracy of the final aggregated records.

Traffic View is another interesting approach described in [2]. It proposes a similar periodic approach based on broadcasting beacons that contained the FCD, like the average speed of a current road segment and its traffic density. The main difference between SOTIS [8] and Traffic View was the computation of the average speed since the aggregation process in Traffic View was an accumulative of average speeds in the road (starting from the quickest vehicle to the slowest one). On the contrary, within SOTIS a vehicle in the center could aggregate all neighboring vehiculars' data within its range. It was blatant that the aggregated record in Traffic View consisted of just one time-stamp value, one position and one speed, as well as vehicular IDs. Hence, this would use better the bandwidth when transmitting messages to every individual vehicle.

CASCADE was suggested in [3, 9] as an optimized Traffic View version [2]. It allowed compressing syntactic data in the aim of optimizing the use of a wireless channel and at the same time guaranteeing accurate aggregated information. Furthermore, it would divide a road into 12 rows $(16 \mathrm{~m} \times$ $126 \mathrm{~m}$ ) of a cluster leading to a $1.5 \mathrm{~km}$ visibility (named a local view). Thus, when vehicles in a local view cluster shared their FCD messages with another cluster, they had an extended view of the whole road segment.

Tsai introduced in [5] a hybrid Aggregating Data Dissemination (ADD) approach that combined both the V2V and V2I models. This approaches also aggregate the number of available free parking spaces in a big region. To do that, the author split a map into a grid structure of square regions. The geodesic distance between these regions was in fact the Road Side Unit (RSU) communication range. The author defined four data aggregation levels in every region. In the first aggregation level, each vehicle would send its parking place, id, position and speed to the RSU center of the region. The RSU would aggregate all received data in the second level before rebroadcasting it to all vehicles in the region. In the third level, the vehicles in the extreme regions would share their traffic information, with the RSU sink. Finally, this later would aggregate, in the fourth level, all information coming from various regions before rebroadcasting it.

Kumar and Dave introduced in [4] a new multi-criteria decision-making for data aggregation. As a matter of fact, the proposed approach assisted a vehicle to decide about the relevance between data, for instance vehicle speed, vehicle direction and free parking space. Hence, the suggested system could decide if two or more input data were similar enough (syntactically or semantically) to be aggregated or not. To achieve this, the authors represented the knowledge base as a KD-tree data structure in order to check the relevance between nodes using the graph characteristics. Although interesting, the provided approach had major drawbacks but mainly it considered the location of aggregates and ignored all other properties (e.g., vehicle directions, maximum allowed speed, etc.). Indeed, this aggregation decision would only consider data coming from the same road segment.

Time aggregated graphs were introduced by George et al. in [10]. They allowed formalizing the road networks and the spatio-temporal properties of the road as a graph data model which would generally support shortest-path query graph algorithms. These time-aggregated graphs could annotate the properties of edges and nodes with the intervals during the time of vehicle presence. However, to decide whatever multiple items could be aggregated or not, the spatio-temporal model was only based on the vehicle travel time, the event life time, and the location. Thus, the major drawback of such a model was that one could not exactly estimate the vehicle travel time, since it could change its direction at any time.

In the same vein, Zeki et al. introduced in [11] an aggregation structure for events produced and exchanged in vehicular networks. More precisely, the proposed structure was based on a spatio-temporal model having two levels. The first one is a physical level consisting of a repository shared between all vehicles to share information without loss. The second one was a logical level where each driver would define his/her 
preferences for what information (s)he was interested in.. This model would manipulate the same shared knowledge base between all vehicles. The ability of being duplicate insensitive and being able to guarantee lossless exchanged information was a significant characteristic of their data structure. In addition, the storage space, mainly needed for the aggregation structure, was particularly limited to include a governable number of temporal dimensions. The main limitation of this solution was the maintenance and the privacy issues of such a shared knowledge base.

In [12], the aggregation of the FCD was only based on the geographical characteristics of the area. For that purpose, the authors introduced the Region-based Location protocol Service Management Protocol (RLSMP) which aimed to reduce the updated positions as well as reducing the number of messages generated to locate car positions. Although the aggregation solution clearly reduced the network overload, it resulted in: i) more packet collisions and consequently more re-transmissions essentially due to the fact that the exchanged packets had a big size, and ii) longer delays owing to the processing carried out on the data.

To sum up, the aggregation process in the aforementioned approaches consists of three phases known as decision, fusion and dissemination:

1) The decision phase, where decision regarding the selection of data items to be fused is made

2) The fusion phase, which is related to the function of fusion. Therefore, all the similar data will be merged in one record

3) The dissemination phase, where aggregated data are broadcasted to other vehicles.

Consequently, the existing aggregation schemes have several limitations, but mainly:

- Security: If the aggregation has the ability to decrease bandwidth consumption problems, it might make security issues harder to manage (e.g., the encryption and the decryption of multiple aggregated and compressed packets) [13].

- Scalability: The existing schemes have medium aggregation time as well as low scalability. This is owing to the fact that when the number of the duplicated exchanged messages goes up, the number of collision problems rises as well [4].

- Genericity: Only few approaches [5, 4] propose a generic model for both aggregation and dissemination mechanisms. In fact, such a combination and synchronisation is of paramount importance to avoid the broadcast storm problem [7] and to decrease the network overhead.

- Filtering: The input items are not fully filtered out (many duplicated items and irrelevant items are not neglected) leading to a high level of network overload.

\section{Smart Directional Data Aggregation PROTOCOL}

Before describing our aggregation protocol, we present in the following subsection several definitions and concepts.

\section{A. Preliminaries}

To better understand our solution, we start by defining the main concepts used in our approach:

Definition 1: Lane (1): It is a one-way path with a paved surface that connects two spatial points on the map. Formally, 1:〈Id, S, E, $\overrightarrow{S E}$, Speed $_{\min }$, Speed $_{\max }$, Status, Location $\rangle$ where:

- Id: represents the identifier of the lane

- $\mathrm{S}$ and $\mathrm{E}$ respectively represent the start and end points which the lane is connecting. Each point is represented by spacial coordinates (e.g., $(\mathrm{x}, \mathrm{y}))$

- $\overrightarrow{S E}$ : represents the directed vector segment from the point $\mathrm{S}$ to $\mathrm{E}$

- Speed $_{\min }$ and Speed $_{\max }$ represent the minimum and maximum speed limits, respectively

- Status: indicates the lane situation (e.g., closed, open, restricted, etc.)

- Location: is the geographical coordinates of the lane in the map.

Definition 2: $\operatorname{Road}(\mathbf{r})$ : It contains at least one or several lanes having the same or different directions. Formally, r: < Id, L, Type, Name, Network Coverage > where:

- Id: represents the road identifier

- L: contains the set of lanes included in the road

- Type: is used to indicate if it is a highway, urban, street, etc.

- Name: is used to describe the road (e.g., street name)

- Network Coverage: indicates the types of network covered within the road (e.g., GSM, WIFI, 3G, 4G, etc.)

Definition 3: Vehicle (v): A vehicle is defined in our approach as follows: v: $\left\langle\right.$ Id, Driving, Speed $_{\max }$, Positioning System, Brand, Type, Size, Environment, DSRC Range, Destination > where:

- Id: represents the vehicle identifier

- Driving: is the set of the driving settings (e.g., Preferred path, Deriving mode: Economic/sport, etc.)

- Speed $_{\max }$ : represents the vehicle maximum speed

- Positioning System: refers to the geographical GISsystem used by the car (e.g., Google Maps, OpenStreetMaps, Bing Maps, etc.)

- Brand: refers to the automobile manufacturer of the vehicle (e.g., BMW, Jeep, Toyota, etc.)

- Type: is used to indicate the type of the car (e.g., MiniVan, Sport, Light Truck, etc.)

- Size: indicates the size of the vehicle

- Environment: refers to the vehicle speed, weather, and the geographic location

- DSRC Range: is the signal power of dedicated wireless short-range communication technology (e.g., 300m, 400m, etc.)

- Destination: is the location to which a vehicle travels.

A vehicle can perform three actions:

- Broadcast: It disseminates fcd messages using the suppression broadcasting technique defined in [7] 
- StoreCarryandForward: A vehicle can store, carry and then rebroadcast the same message using the rebroadcasting technique defined in [7]

- CalculateAverageSpeed: It calculates the average speed using the aggregation function defined in section III-B

- Receive: It receives all types of messages sent using the DSRC protocol [1]

- CalculateDirection: A vehicle is able to determinate its direction using a positioning system (e.g., Google Maps) based on its location and its destination

- LocateLane: A vehicle is able to locate the current driving lane based on its geographic location.

In our solution, we assume that our aggregation protocol runs on the top of the MAC-layer, so it requires no modification in IEEE $802.11 \mathrm{p}$ standard [1]. Also, we acquire that only one type of messages can be generated and sent, namely the Floating Car Data (FCD) known also as Floating Cellular Data [8].

Definition 4: FCD message (f): The adopted FCD messages header structure is therefore defined as a 4-tuple: $\mathrm{f}$ : 〈 SenderId, SenderPosition, AverageSpeed, Destination \rangle where:

- SenderId: is the unique identifier of vehicle that sends the message

- SenderPosition: contains the spatial position of the sender

- AverageSpeed: is the average speed (computed using a function defined in Section III-B)

- Destination: contains the future location of the vehicle sending the FCD message.

The size of the message is less than 2,321 Bytes, which is the maximal allowed size as defined by $802.11 \mathrm{p}$ standard [1].

\section{B. Aggregation protocol}

Our aggregation algorithm deals with the following three scenarios: a unidirectional road, a bidirectional road, and an urban scenario. The aggregation function used in our protocol is the same average function used in SOTIS [8]. We choose the main average speed aggregation function of SOTIS since our contribution can be seen as an optimisation of SOTIS. However, as mentioned in the related work, only three approaches, SOTIS [8], TrafficView [2] and CASCADE [9], focus only on the aggregation of the vehicles speed without combining other driver preferences. The average speed aggregation function of SOTIS is defined as follows [8]:

$$
\hat{V}_{r, \text { new }}=\hat{V}_{r, \text { prev }}+\hat{V}_{r}
$$

where $\hat{V}_{r, \text { new }}$ is the new average speed for the road $\mathrm{r}, \hat{V}_{r, \text { prev }}$ is its previous average speed, and $\hat{V}_{r}$ stands for the average speed of the vehicles on the road $r$. Each vehicle has three aggregation cases: unidirectional-road, bidirectional-road and urban-city. Algorithm 1 depicts the behavior of a vehicle $v$ upon receipt of an FCD message.

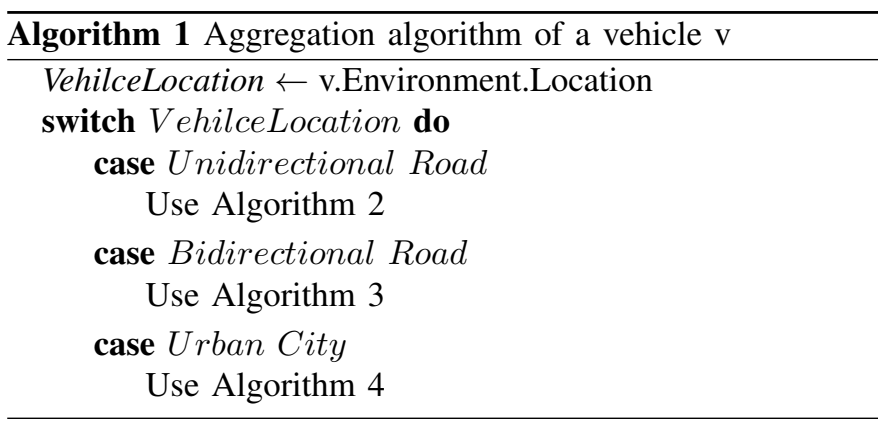

1) Unidirectional Road Case: On an unidirectional road, all vehicles drive in the same direction. Indeed, vehicles ahead must collect traffic information, aggregate and disseminate it to other vehicles located behind. Figure 1 depicts the unidirectional road scenario where lanes contain different speed limitations. Algorithm 2 contains the pseudo-code of processing this case. Briefly, when a vehicle $v$ receives an FCD

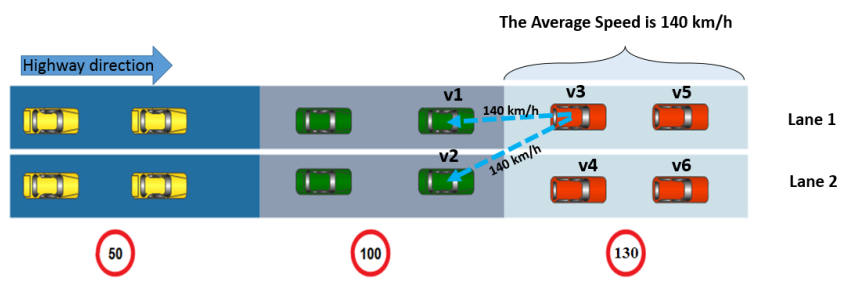

Fig. 1. Unidirectional Road Case

message, it checks if the message comes from a farther sender on the same road or not. If yes, it computes the new updated average speed $\hat{V}_{r, \text { new }}$ based on Equation 1. If the received average speed is greater than the maximum allowed speed of the current road $r$, the vehicle receiving the message will keep its previous average speed $\hat{V}_{r, p r e v}$ and rebroadcast the value of the road maximum speed. Doing so, the propagated FCD message will warn other vehicles' drivers that ahead vehicles are driving very fast and exceed the maximum allowed speed. In Figure 1, only vehicles $v_{1}$ and $v_{2}$ will receive and deal with the FCD message sent form vehicle $v_{3}$ since they are in the broadcast range and drive behind it. However, vehicles $v_{1}$ and $v_{2}$ will ignore the received average speed, $140 \mathrm{~km} / \mathrm{h}$, and broadcast the road maximum allowed speed (of $130 \mathrm{~km} / \mathrm{h}$ ). Doing so, all the disseminated traffic information will follow the legal speed.

2) Bidirectional Road Case: On a bidirectional road, vehicles drive in two opposite directions. Indeed, vehicles in the opposite directions must collect traffic information, and then aggregate and disseminate it to other opposite vehicles as shown in Figure ??. The exchange of traffic information between the two opposite directions has a paramount importance. In essence, such an exchange would inform and warn all drivers about the traffic conditions ahead, which leads to avoid traffic jam and road accidents. Figure 2 illustrates the bidirectional road scenario where lanes contain different speed limitations. Indeed, vehicle $v_{4}$ will share its FCD average 


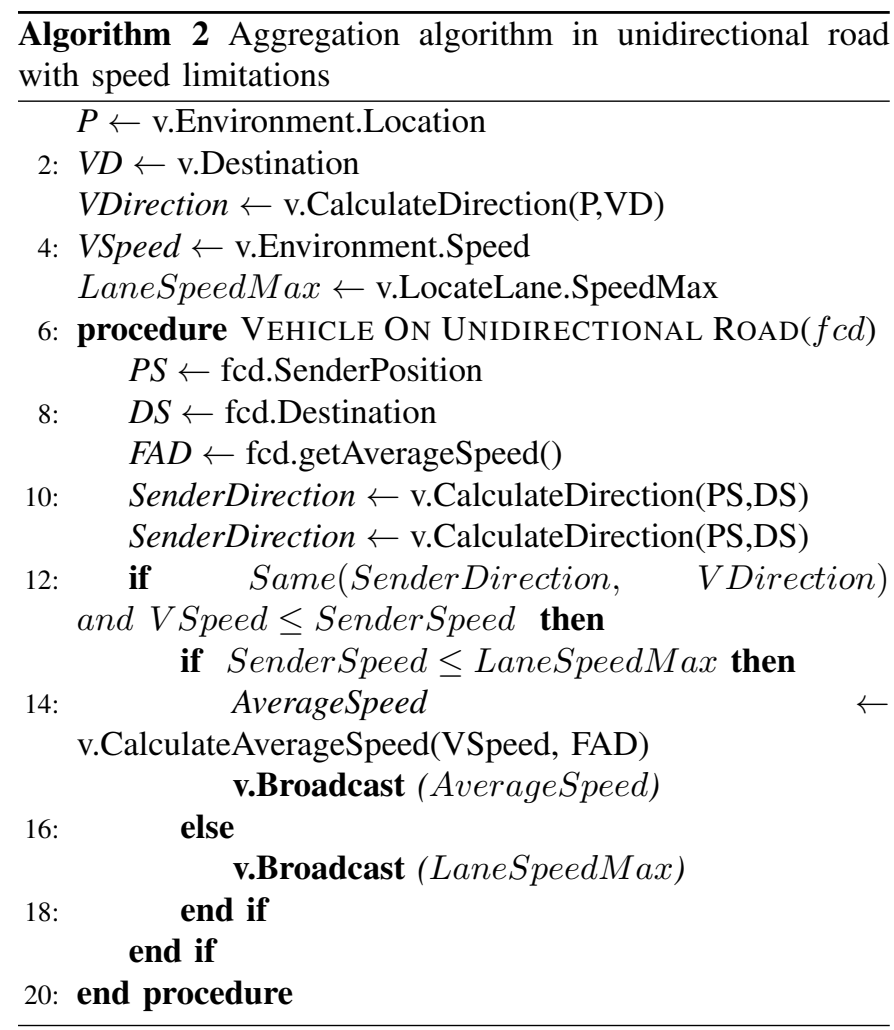

speed $(90 \mathrm{~km} / \mathrm{h})$ with vehicle $v_{1}$, which will broadcast this information to other behind vehicles since the average speed is less than the maximum allowed speed. Algorithm 3 contains the pseudo-code of processing this case. In fact, the main difference between this case and the previous one is that in a bidirectional scenario, vehicles can accept FCD messages that come form the opposite side or vehicles ahead in the same lane and ignore other messages that come from behind. Actually,

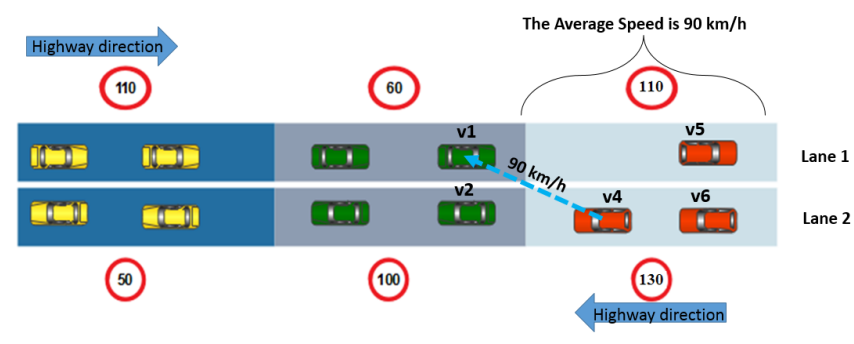

Fig. 2. Bidirectional road case

the vehicles in the opposite side have a larger overview on the opposite traffic conditions, since they have been passed in front of it. Doing that allows guaranteeing that the FCD contains an aggregated value of the whole opposite lanes.

3) Urban Network Case: In urban network, vehicles drive in many different directions. Thus, vehicles can meet over cross roads and junctions. Indeed, they must exchange their traffic information ,as shown in Figure 3. However, using a blind aggregation method will affect the precision and the accuracy of the exchanged traffic information. This is owning to the fact that vehicles can share and aggregate the average speed of other vehicles that are not going to the same direction. Our aggregation model can solve this issue based on three filters:
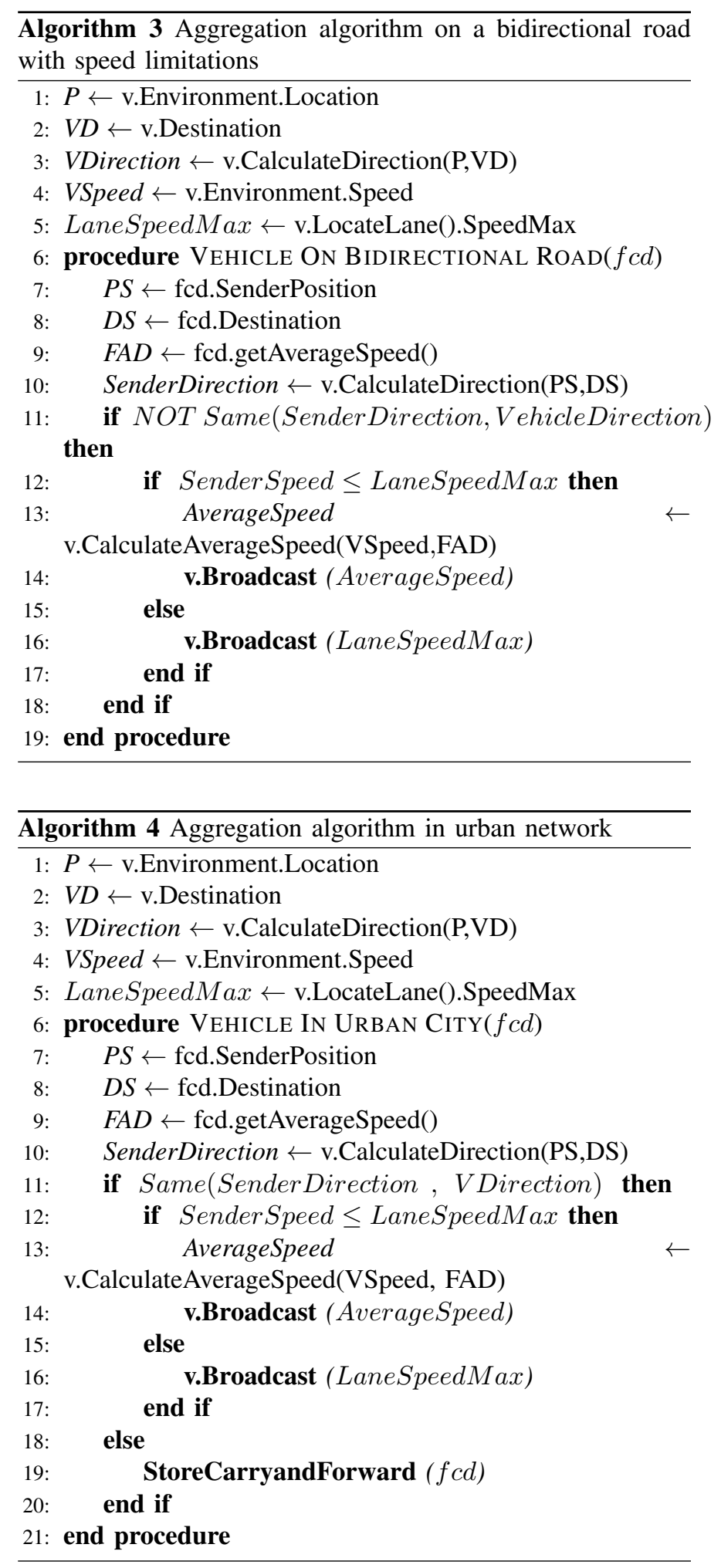

1) A direction filter that ensures the aggregation of coherent FCD messages targeting the same road and direction 
2) A suppression technique filter that ignores all duplicated FCD messages. In the current version of our protocol, we adopt the famous slotted 1-persistence broadcast protocol [7]

3) An aggregation filter that ignores all the received FCD messages that exceed the road maximum speed. In fact, if the average speed of any FCD message is greater than the maximum allowed speed, the received vehicle will ignore it and broadcast the maximum speed instead.

In urban networks, when a vehicle gets an FCD message, it checks firstly the direction of the sender. Then if the received average speed is less than the road maximum speed limit, the vehicle will aggregate and disseminate the received FCD message. Otherwise, the received message will be ignored. Indeed, when a vehicle $v$ receives a message, not in its

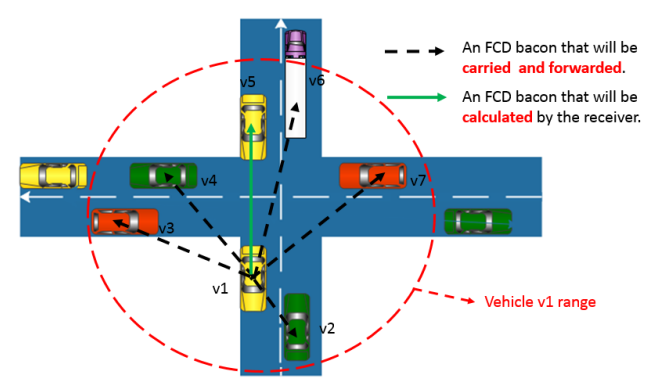

Fig. 3. Urban scenario case

direction, it will store, carry, and then forward it to other vehicles that may be going to this direction. Doing so, the traffic information will be disseminated to all vehicles in the road intersections. Algorithm 4 depicts a vehicle behavior upon receiving an FCD message in an urban situation.

\section{EXPERIMENTATIONS}

In this section, we present the performance evaluation conducted to evaluate our aggregation protocol. We choose SOTIS [8] and TrafficView [2] as baseline approaches since they focus only on the aggregated FCD without combining other data (like safety and non-safety events). CASCADE [3] will not be considered in our evaluation since it uses a compression algorithm on the top of TrafficView [2] to optimize the MAC-Layer utilization (this is out of scope of our study here but can also be applied by our approach after aggregation). We carry out our experiments using the Veins simulator framework ${ }^{1}$. Veins is an open source framework for Inter-Vehicular Communication (IVC) that combines both a road traffic micro-simulation model and an event-based network simulator. To conduct the experiments, we choose the default road map of the city of Manhattan in the framework. This city has a very high complex road topology that contains both urban and highway networks. We vary the amount of vehicles driving on the map from 200 to 1000, ranging from low traffic to high traffic.

\footnotetext{
${ }^{1}$ http://veins.car2x.org/
}

\section{A. Experimental settings}

The road traffic simulation is performed by SUMO ${ }^{2}$ while the network simulation is performed by OMNeT++ along with the physical layer modeling toolkit $\mathrm{MiXiM}^{3}$, allowing the employment of accurate models for radio interference, as well as shadowing the use of static and moving obstacles. With these two well-established simulators, nodes simulated by OMNeT++ 5.1.0 can interact with SUMO to simulate the influence of IVC on road traffic and mobility. We take advantage of these two simulators included in Veins to provide realistic models for 802.11p DSRC, PHY and MAC layers. The PHY and MAC parameters are defined according to the basic specifications of the 802.11p standard defined in [14]. In the MAC layer, we set the transmission power of a vehicle to $30 \mathrm{~mW}$ to achieve approximately $300 \mathrm{~m}$ of interference range

\section{B. Evaluation metrics}

The assessment of the performances of our protocol is carried out through the following metrics:

1) Overload: It stands for the total number of sent packets. Interestingly enough, the ultimate goal of any aggregation protocol is to avoid the overload problem [1] by looking for minimizing the number of messages exchanged in the network. The average overload is defined as follows:

$$
\text { Overload }=\frac{\sum \text { sent packet }}{\sum \text { vehicle }}
$$

2) Co2Emission: refers to the amount of $\mathrm{Co} 2$ getting out from vehicles and affecting the environment [15]. Indeed, the ultimate goal of any traffic information system, and especially any aggregation protocol, is to reduce the total $\mathrm{Co} 2$ emmision [15] by looking for minimizing as much as possible traffic jams and decreasing the active waiting time of vehicles in cross roads and on highways. The average $\mathrm{Co} 2$ emission is defined as follows:

$$
\text { Co2Emission }=\frac{\sum \text { Vehicle Co2Emission }}{\sum \text { vehicle }}
$$

3) Latency: It is the time needed to deliver the aggregated message to an interested vehicle. The average latency, $A L$, is defined as follows:

$$
A L=\frac{\sum\left(t_{v_{i}}-T\right)}{\sum \text { Interested Vehicle }}
$$

where $t_{i}$ stands for the arrival time of the event message to a vehicle $v_{i}$, and $T$ is the time-stamp of the event.

4) FCD Duplication Ratio: It is the number of messages that are already sent and aggregated for a given message $f$. It is defined as follows:

$$
\text { Duplication ratio }=\frac{\sum \text { Duplicated } f}{\sum f}
$$

${ }^{2}$ http://http://sumo.dlr.de

${ }^{3}$ http://mixim.sourceforge.net/ 


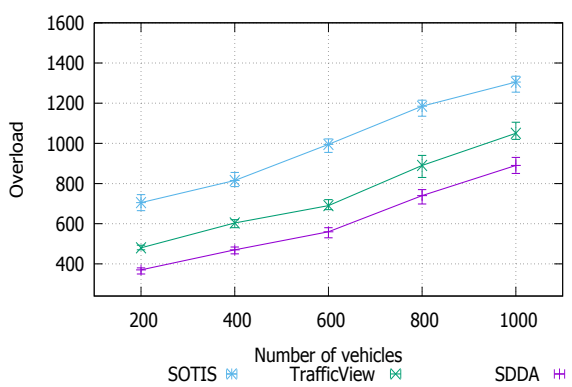

Average overload (a)

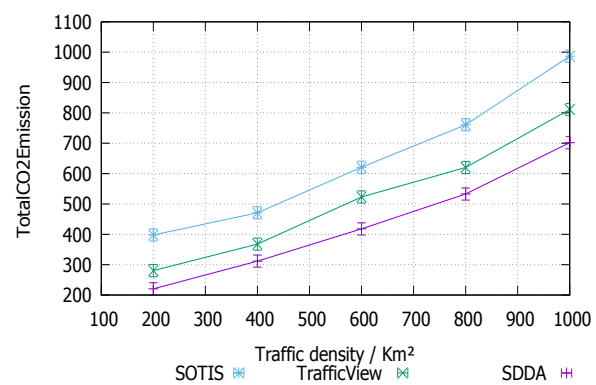

Average Co2Emission (b)

Fig. 4. Variation of average overload/Co2 emission values w.r.t. number of vehicles

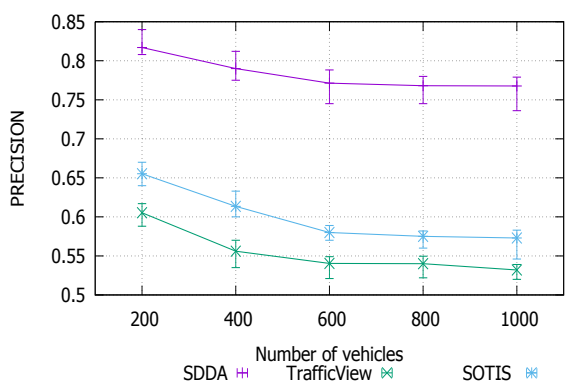

Aggregation precision (a)

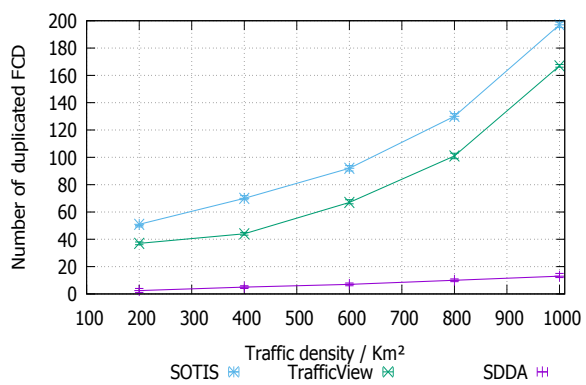

Number of duplicated FCD (b)

Fig. 5. Variation in average aggregation precision/Number of duplicated FCD values w.r.t. number of vehicles

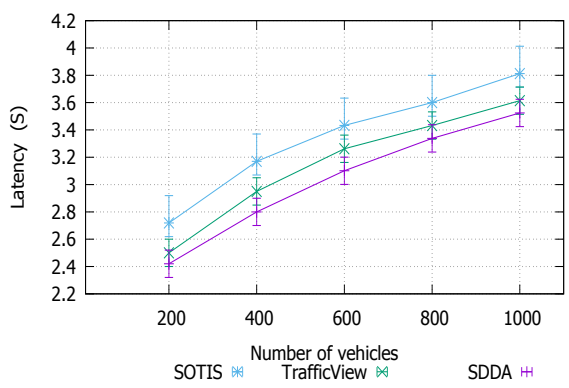

Average Latency (a)

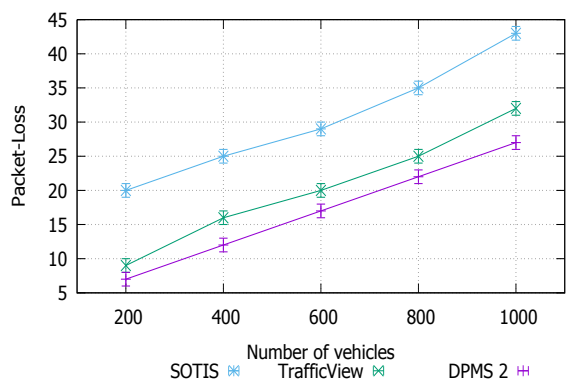

Packet loss (b)

Fig. 6. Variation of average latency/packet loss values w.r.t. number of vehicles

5) Aggregation Precision: It assesses to what extent our aggregation protocol is able to only aggregate an appropriate FCD message $f$ for a given road $r$ without duplication and to take into consideration the road maximum speed. Hence, the challenge will be to obtain precision values of the average speed propagated to vehicles compared to the real road traffic state. It is defined as follows:

$$
\operatorname{Precision}(f)=\frac{|I I V|}{|A I V|}
$$

where $I I V$ stands for the set of interested informed vehicles (i.e., only appropriate vehicles for a message $f$ ), and $A I V$ stands for the set of all informed vehicles that will aggregate the received FCD message $f$ (i.e., interested as well as not interested vehicles for an FCD $f)$. The average precision is defined as follows:

$$
\text { AveragePrecision }=\frac{\sum \text { Precision }(f)}{\sum f}
$$

\section{Results}

Figure 4 shows the evolution of the overload and co2emission values in different density networks. As expected, using our aggregation protocol, the overload level is decreased (cf. Figure 4-a). This is owed to the fact that the number of duplicated messages is eliminated and the propagation of unnecessary aggregated FCD messages to uninterested vehicles is reduced. As a result, our protocol SDDA enables keeping a low overload. Moreover, Figure 4-b shows that SDDA has a low Co2 Emmision value in different network densities. It is worth mentioning that our protocol decreases the co2Emmision value in comparison to SOTIS and TrafficView by almost $70 \%$ since 
vehicles will have a precise traffic information and will avoid traffic jams. Figure 4 also demonstrates that the overload and co2Emmision values will rise for all protocols when the number of vehicles increases. This interesting performance is related to the fact that our strategy permits ignoring all the unnecessary FCD messages when vehicles move based on direction and speed limitation filers. Moreover, Figure 5$\mathrm{b}$ indicates that the number of duplicated messages in our proposed solution is $99 \%$ less than other solutions thanks to the usage of the Slotted-1 persistence suppression technique [7] being able to eliminate all the duplicated messages. Finally, Figure 6-a depicts that the latency of our SDDA strategy is slightly less than that of other strategies. This is due to the high overload level of SOTIS and TrafficView which increase the network collisions that affects the latency time. As expected, the packet loss ratio in Figure 6-b is inversely proportional to the traffic density. In fact, it decreases as far as the number of vehicles grows. This can simply be explained by the fact that when raising the number of vehicles in the network, the communication overload as well as the message reception errors go up, which leads to increase the ratio of lost packets. Consequently, this fact will undoubtedly increase the number of vehicles that will not receive the messages or will receive damaged packets (unreadable, corrupted, etc.). To sum up, the simulation results highlight that our strategy performs much better than the baseline strategies from different perspectives (overload, co2emission, aggregation precision, packet loss and latency). Also, it is important to note that our strategy is genetic and can be adapted to any other aggregation context like road conditions, commercial advertisements and trip travel time.

\section{CONCLusion}

In this paper, we have introduced SDDA as a generic smart directional data aggregation model protocol to exchange information about traffic in a VANET to overcome several limitations related to existing approaches. The main thrust of our protocol stands for an adequate targeting of using direction and road speed limitation. Doing so has allowed meeting several goals, namely reaching a low overload ratio as well as a high aggregation precision. Extensive experimental work has shown that SDDA obtains very interesting results in comparison with those provided by pioneering approaches of the literature. Avenues of future work are as follows:1)We are actually working on real-word validation scenarios and we plan to extend the model to deal with other aggregation issues in VANET like parking spaces, road conditions, and trip travel time, 2) Provide a generic compression algorithm for FCD messages in order to reduce the bandwidth usage, and 3) Integrate other dissemination protocols in our aggregation model in order to provide a more generic aggregation and dissemination protocol.

\section{REFERENCES}

[1] F. C. Commission, "Amendments of the commission's rules regarding dedicated short-range communication ser- vices in the $5.9 \mathrm{ghz}$ band," Federal Communications Commission Proceedings, pp. 10-809, 2004.

[2] T. Nadeem, S. Dashtinezhad, C. Liao, and L. Iftode, "Trafficview : Traffic data dissemination using car-to-car communication," ACM SIGMOBILE Mobile Computing and Communications Review, no. 3, pp. 1-8, 2004.

[3] K. Ibrahim and M. C. Weigle, "Cascade: Cluster-based accurate syntactic compression of aggregated data in vanets," 2008 IEEE Globecom Workshops, GLOBECOM 2008, pp. 1-10, 2008.

[4] R. Kumar and M. Dave, "A framework for handling local broadcast storm using probabilistic data aggregation in vanet," Wireless Personal Communications, p. 315-341, 2013.

[5] H. W. Tsai, "Aggregating data dissemination and discovery in vehicular ad hoc networks," Telecommunication Systems, p. 285-295, 2013.

[6] Y. Zhu, Q. Zhao, and Q. Zhang, "Delay-constrained data aggregation in vanets," IEEE Transactions on Vehicular Technology, pp. 2097-2107, 2015.

[7] N. Wisitpongphan, O. Tonguz, J. Parikh, P. Mudalige, F. Bai, and V. Sadekar, "Broadcast storm mitigation techniques in vehicular ad hoc networks," Wireless Communications, IEEE, vol. 14, no. 6, pp. 84-94, 2007.

[8] L. Wischoff, A. Ebner, H. Rohling, M. Lott, and R. Halfmann, "Sotis - a self-organizing traffic information system," in The 57th IEEE Semiannual Vehicular Technology Conference, 2003. VTC 2003-Spring., 2003, pp. 1-6.

[9] K. Ibrahim, "Data aggregation and dissemination in vehicular ad-hoc networks by data aggregation and dissemination in vehicular ad-hoc networks," no. 4, pp. 1120,2011

[10] B. George and S. Kim, "Spatio-temporal networks," Database Management and Information Retrieval, 2013.

[11] D. Zekri, B. Defude, and T. Delot, "Building , sharing and exploiting spatio- temporal aggregates in vehicular networks," Hindawi Mobile Information Systems, vol. 14, pp. 259-285, 2014.

[12] H. Saleet, R. Langar, K. Naik, R. Boutaba, A. Nayak, and N. Goel, "Intersection-based geographical routing protocol for vanets: A proposal and analysis," IEEE Transactions on Vehicular Technology, pp. 4560-4574, 2011.

[13] S. Mohanty and D. Jena, "Secure data aggregation in vehicular-adhoc networks: A survey," 2nd International Conference on Communication, Computing and Security ICCCS-2012, vol. 6, pp. 922 - 929, 2012.

[14] C. Sommer, R. German, and F. Dressler, "Bidirectionally coupled network and road traffic simulation for improved ivc analysis," IEEE Transactions on Mobile Computing, vol. 10, pp. 3-15, 2011.

[15] A. Cappiello, I. Chabini, E. K. Nam, A. Lue, and M. Abou Zeid, "A statistical model of vehicle emissions and fuel consumption," IEEE 5th Conference on Intelligent Transportation Systems, pp. 801-809, 2002. 